\title{
Riscos e benefícios dos diferentes métodos de introdução alimentar
}

\author{
Risks and benefits of different methods of complementery feeding \\ Riesgos y beneficios de los diferentes métodos de introducción de alimentos
}

Recebido: 25/03/2021 | Revisado: 03/04/2021 | Aceito: 08/04/2021 | Publicado: 17/04/2021

\author{
Camila Conceição Santos Temóteo \\ ORCID: https://orcid.org/0000-0002-6469-2996 \\ Universidade Tiradentes, Brasil \\ E-mail: camilacstemoteo@gmail.com \\ Débora Cristina Leite Fontes \\ ORCID: https://orcid.org/0000-0002-5396-2428 \\ Universidade Tiradentes, Brasil \\ E-mail: deboraleite2006@hotmail.com \\ Alessandro Santos Ferreira \\ ORCID: https://orcid.org/0000-0003-1781-4759 \\ Universidade Tiradentes, Brasil \\ E-mail: alessandro180598@gmail.com
}

\begin{abstract}
Resumo
Introdução: Os primeiros anos de vida de uma criança são caracterizados por rápida velocidade de crescimento e desenvolvimento digestório e neuropsicomotor, incluindo habilidades tais como: receber, mastigar e digerir outros alimentos além do leite materno. Objetivo: compreender se há dentre os diferentes métodos de alimentação complementar algum que ofereça maior risco ou benefícios para saúde, desenvolvimento e segurança do bebê. Metodologia: foram utilizados os descritores Complementary feeding AND Baby led weaning AND Infant nutrition. Segundo o mecanismo Medical Subject Headings (MeSH) e Descritores em Ciências em Saúde (DeCS), das três termologias apenas uma, Infant Nutrition, consta no vocabulário controlado. Dentre os 105 artigos encontrados, 71 da US National Library of Medicine (PubMed) e 34 da Biblioteca Virtual em Saúde (BVS), 23 estavam presentes em ambas as bases de dados, 21 artigos foram lidos de forma integral e 8 foram elegíveis para o presente trabalho Resultados e discussão: Foram analisados 8 trabalhos para presente revisão, dos quais 6 são ensaios clínicos randomizados, 1 estudo piloto realizado através de questionário e 1 estudo realizado através de questionário online. Além dos valores baseados na pesagem da dieta, aos 12 meses os bebes passaram por uma avaliação laboratorial através da coleta de sangue. Os valores para hemoglobina (g/dl) no grupo controle foi de 11,7 e no grupo BLISS 11,6. Conclusão: Está revisão não identificou diferenças significativas entres os diferentes métodos de introdução alimentar. Palavras-chave: Alimentação complementar; BLW; Nutrição infantil; Aleitamento materno; Engasgo.
\end{abstract}

\begin{abstract}
Introduction: The first years of a child's life are characterized by rapid growth and digestive and neuropsychomotor development, including skills such as: receiving, chewing and digesting foods other than breast milk. Objective: to understand if there are any of the different complementary feeding methods that offer greater risk or benefits for the baby's health, development and safety. Methodology: Complementary feeding AND Baby led weaning AND Infant nutrition were used. According to the Medical Subject Headings (MeSH) and Descriptors in Health Sciences (DeCS), of the three thermologies, only one, Infant Nutrition, appears in the controlled vocabulary. Among the 105 articles found, 71 from the US National Library of Medicine (PubMed) and 34 from the Virtual Health Library (VHL), 23 were present in both databases, 21 articles were read in full and 8 were eligible for the present work Results and discussion: 8 papers were analyzed for this review, of which 6 are randomized clinical trials, 1 pilot study conducted through a questionnaire and 1 study conducted through an online questionnaire. In addition to the values based on the weighing of the diet, at 12 months the babies underwent a laboratory evaluation through blood collection. The values for hemoglobin $(\mathrm{g} / \mathrm{dl})$ in the control group was 11.7 and in the BLISS group 11.6. Conclusion: This review did not identify significant differences between the different methods of food introduction.
\end{abstract}

Keywords: Complementary feeding; BLW; Child nutrition; Breast feeding; Gagging.

\section{Resumen}

Introducción: Los primeros años de vida de un niño se caracterizan por un rápido crecimiento y desarrollo digestivo y neuropsicomotor, incluyendo habilidades como: recibir, masticar y digerir alimentos distintos a la leche materna. Objetivo: conocer si existe alguno de los diferentes métodos de alimentación complementaria que ofrezcan mayor riesgo o beneficios para la salud, el desarrollo y la seguridad del bebé. Metodología: Se utilizaron alimentación complementaria Y destete dirigido por bebés Y Nutrición infantil. De acuerdo con el mecanismo de Medical Subject 
Headings (MeSH) y descriptores en ciencias de la salud (DeCS), de las tres termologías solo una, Nutrición infantil, aparece en el vocabulario controlado. Entre los 105 artículos encontrados, 71 de la Biblioteca Nacional de Medicina de EE. UU. (PubMed) y 34 de la Biblioteca Virtual en Salud (BVS), 23 estaban presentes en ambas bases de datos, 21 artículos fueron leídos en su totalidad y 8 fueron elegibles para el presente trabajo Resultados y discusión: se analizaron 8 artículos para esta revisión, de los cuales 6 son ensayos clínicos aleatorizados, 1 estudio piloto realizado a través de un cuestionario y 1 estudio realizado a través de un cuestionario en línea. Además de los valores basados en el pesaje de la dieta, a los 12 meses los bebés se sometieron a una evaluación de laboratorio mediante la recogida de sangre. Los valores de hemoglobina ( $\mathrm{g} / \mathrm{dl}$ ) en el grupo de control fueron 11,7 y en el grupo BLISS 11,6. Conclusión: esta revisión no identificó diferencias significativas entre los diferentes métodos de introducción de alimentos.

Palabras clave: Alimentación complementaria; BLW; Nutrición infantil; Lactancia materna; Atragantamiento.

\section{Introdução}

Os primeiros anos de vida de uma criança são caracterizados por rápida velocidade de crescimento e desenvolvimento digestório e neuropsicomotor, incluindo habilidades tais como: receber, mastigar e digerir outros alimentos além do leite materno (SBP,2017). A qualidade e a quantidade de alimentos consumidos pela criança são aspectos críticos e têm repercussões ao longo de toda a vida, associando-se ao perfil de saúde e nutrição, já que a infância é um dos estágios da vida biologicamente mais vulnerável às deficiências e aos distúrbios nutricionais (Lopes et al., 2018).

A nutrição infantil inadequada eleva o risco para o desenvolvimento de enfermidades (Sankar et al., 2015). Dentre os fatores determinantes para à boa saúde, o de maior impacto é a alimentação adequada durante a infância, principalmente nos seus dois primeiros anos de vida (Victora et al., 2016). A OMS recomenda que, aos seis meses de idade, deve-se iniciar a introdução de alimentos complementares apropriados, seguros e nutricionalmente adequados (WHO,2001). Devem ser apresentados em quantidade e consistência própria para a idade da criança e sem excessos de sal e condimentos (Lima et al., 2012).

Alimentação complementar é definida como a alimentação no período em que outros alimentos ou líquidos são oferecidos à criança, em adição ao leite materno (Lima et al.,2012). A introdução da alimentação complementar é um momento muito sensível, de fundamental relevância para a saúde da criança (Sorrentino,2018). Segundo o Manual de orientação do departamento de nutrologia seguindo o modelo tradicional de alimentação complementar após os 6 meses a alimentação deve ser espessa e oferecida com colher, iniciando com uma consistência pastosa e progredindo gradativamente a consistência até chegar à alimentação da família (SBP, 2012).

Além das orientações publicadas por comitês profissionais, outras formas de alimentação complementar estão sendo difundidas, dentre elas o Baby-Led Weaning (BLW), termo que tem por significado: o desmame guiado pelo bebê, conceito idealizado pela britânica Gill Rapley (SBP, 2012). Outro método de alimentação complementar é o Baby-Led introduction to SolidS (BLISS). Essa abordagem foi proposta por um grupo de estudiosos neozelandeses com o propósito de sanar questionamentos levantados por profissionais e pais quanto ao risco de engasgo e baixa oferta de ferro e calorias que poderia advir do método BLW (Fangupo LJ et al.,2016). No entanto, há muitas dúvidas aos potenciais reflexos no comportamento alimentar e no crescimento/ desenvolvimento (Andries e Arantes et al., 2018).

Diante do exposto, a presente revisão tem por objetivo compreender se há dentre os diferentes métodos de alimentação complementar algum que ofereça maior risco ou benefício para saúde, desenvolvimento e segurança do bebê.

\section{Metodologia}

Realizou-se uma pesquisa preliminar para reconhecimento do tema da presente revisão, verificou-se que em algumas bases de dados referências de interesse ao trabalho eram escassas. Por tanto, optou-se por utilizar a Biblioteca Virtual em Saúde (BVS) e o US National Library of Medice (PubMed), bases que possuem notoriedade na área da saúde. Foram utilizados 
os descritores Complementary feeding AND Baby led weaning AND Infant nutrition. Segundo o mecanismo Medical Subject Headings (MeSH) e Descritores em Ciências da Saúde (DeCS), das três termologias apenas uma, Infant Nutrition, consta no vocabulário controlado. Porém, para o presente trabalho julgou-se de fundamental importância utilizar as demais, visto que as referências de interesse citavam ao menos uma das terminologias no título e/ou palavras-chaves.

Os métodos abordados na pesquisa foram o método tradicional, o qual é orientado pela OMS, o BLW e o BLISS, caracterizado também como BLW modificado.

A busca eletrônica foi realizada do mês de julho ao mês de agosto de 2020 e encontrou-se um total de 105 artigos por meio de busca nas bases de dados, US National Library of Medice (PubMed) e Biblioteca Virtual em Saúde (BVS). Foi posta restrição em relação ao período de publicação, sendo o período estabelecido dos anos 2005 a 2020, sendo o ano inicial determinado pelo período de início das discussões a respeito do método BLW (Rapley,2015). Os critérios de inclusão estabelecidos foram: estudos nos idiomas inglês e espanhol provenientes de ensaios clínicos randomizados, ensaios clínicos controlados e estudos observacionais que abordaram o tema da alimentação complementar e os diferentes métodos os quais trazem alguma perspectiva a respeito dos benefícios e possíveis riscos dos tais. Como critérios de exclusão estabelecidos foram: artigos de revisão, editoriais, cartas ao editor e artigos não disponíveis na íntegra.

A presente revisão possui uma abordagem quantitativa (De-la-Torre-Ugarte-Guanilo MC et al., 2011), realizada em quatro etapas: (I) Formulação dos questionamentos: há risco e benefícios dentre os diferentes métodos de introdução alimentar? Se há, quais são? (II) Busca e seleção dos artigos nas bases de dados de escolha. (III) Exclusão e inclusão dos artigos de acordo com os critérios estabelecidos. (IV) Extração dos dados, a partir de avaliação minuciosa dos artigos selecionados.

Dentre os 105 artigos encontrados, 71 da US National Library of Medicine (PubMed) e 34 da Biblioteca Virtual em Saúde (BVS), 23 estavam presentes em ambas as bases de dados, 21 artigos foram lidos de forma integral e 8 foram elegíveis para o presente trabalho.

\section{Resultados e Discussão}

Foram analisados 8 trabalhos para presente revisão, dos quais 6 são ensaios clínicos randomizados, 1 estudo piloto realizado através de questionário e 1 estudo realizado através de questionário online. As principais temáticas abordadas foram o consumo energético, ingesta de nutrientes e risco de engasgo. 
Tabela 1: Características dos estudos incluídos na revisão. Aracaju, SE, 2020.

\begin{tabular}{|c|c|c|c|c|}
\hline Autor (ano) & País & Amostra & Métodos avaliados & Principais resultados \\
\hline $\begin{array}{l}\text { Taylor et al. } \\
(2017)\end{array}$ & $\begin{array}{l}\text { Nova } \\
\text { Zelândia }\end{array}$ & $\begin{array}{l}206 \text { crianças, } 101 \text { do } \\
\text { grupo controle e } 105\end{array}$ & $\begin{array}{l}\text { Método tradicional } \\
\text { e BLISS }\end{array}$ & $\begin{array}{l}\text { IMC aos } 24 \text { meses: Controle: 16,2; } \\
\text { BLISS: } 16,4\end{array}$ \\
\hline & & BLISS & & $\begin{array}{l}\text { Consumo energético aos } 7 \text { meses: } \\
\text { Controle (KJ): } 2862 \text {; BLISS: } 2996 .\end{array}$ \\
\hline $\begin{array}{l}\text { Morison et al. } \\
(2016)\end{array}$ & $\begin{array}{l}\text { Nova } \\
\text { Zelândia }\end{array}$ & $\begin{array}{l}51 \text { crianças, } 26 \text { Método } \\
\text { tradicional, } 7 \quad \text { BLW } \\
\text { parcial e } 18 \text { BLW Total }\end{array}$ & $\begin{array}{l}\text { Método Tradicional } \\
\text { e BLW }\end{array}$ & $\begin{array}{l}\text { Consumo energético (KJ): MT: 2897; } \\
\text { BLW parcial: } 3073 \text { e BLW total } 2800 \text {. } \\
\text { Ingesta de Zinco (mg): MT: } 3,7 \mathrm{mg} \text {; BLW } \\
\text { parcial: 4,0; BLW total: 3,0. } \\
\text { Ingesta de Ferro (mg): MT: 3,6; BLW } \\
\text { parcial: 3,3 e BLW total: } 1,6 \text {. }\end{array}$ \\
\hline Dogan et al. (2016) & Turquia & $\begin{array}{l}280 \text { bebês, } 138 \text { Método } \\
\text { tradicional e } 142 \text { BLW }\end{array}$ & $\begin{array}{l}\text { Método tradicional } \\
\text { e BLW }\end{array}$ & $\begin{array}{l}\text { Média do peso aos } 12 \text { meses }(\mathrm{Kg}) \text { : BLW: } \\
\text { 10,4 e MT: } 11,1 . \\
\text { Ingesta de Ferro aos } 12 \text { meses }(\mathrm{mg} / \mathrm{dia}) \text { : } \\
\text { BLW: } 7,97 \text { e MT: } 7,90 . \\
\text { Episódios de engasgo: } \\
\text { BLW: } 2 \text { e MT: } 3 \text {. }\end{array}$ \\
\hline $\begin{array}{l}\text { Cameron et al. } \\
(2015)\end{array}$ & $\begin{array}{l}\text { Nova } \\
\text { Zelândia }\end{array}$ & $\begin{array}{l}23 \text { participantes, } 14 \\
\text { BLISS e } 9 \text { BLW }\end{array}$ & BLISS e BLW & $\begin{array}{l}\text { Valor energético fornecido na dieta } \\
\text { complementar (KJ/dia): } \\
\text { BLISS: } 2228 \text { e BLW: } 1862 . \\
\text { Episódios de engasgo: } \\
\text { BLISS: } 2 \text { e BLW: } 1 .\end{array}$ \\
\hline $\begin{array}{l}\text { Erickson et } \\
(2018)\end{array}$ & $\begin{array}{l}\text { Nova } \\
\text { Zelândia }\end{array}$ & $\begin{array}{l}206 \text { participantes, } 101 \\
\text { Controle e } 105 \text { BLISS. }\end{array}$ & $\begin{array}{l}\text { Método tradicional } \\
\text { e BLISS }\end{array}$ & $\begin{array}{l}\text { Consumo energético (KJ): } \\
\text { - Aos } 7 \text { meses: } \\
\text { Controle: } 2831 \text {; BLISS: } 2951 \\
\text { - Aos } 12 \text { meses: } \\
\text { Controle: } 3373 \text {; BLISS: } 3484 \\
\text { - Aos } 24 \text { meses: } \\
\text { Controle: } 4003 \text {; BLISS: } 3982\end{array}$ \\
\hline $\begin{array}{l}\text { Daniels et } \quad \text { al. } \\
(2018)\end{array}$ & $\begin{array}{l}\text { Nova } \\
\text { Zelândia }\end{array}$ & $\begin{array}{l}206 \text { participantes, } 101 \\
\text { Controle e } 105 \text { BLISS }\end{array}$ & $\begin{array}{l}\text { Metodo Tradicional } \\
\text { e BLISS }\end{array}$ & $\begin{array}{l}\text { Hemoglobina }(\mathrm{g} / \mathrm{dl}) \text { : } \\
\text { Controle: } 11,7 \text { e BLISS: } 11,6 \text {; } \\
\text { Ferritina plasmática }(\mu / \mathrm{dl}) \text { : } \\
\text { Controle: } 28,9 \text { e BLISS: } 27,0\end{array}$ \\
\hline $\begin{array}{l}\text { Brow A. } \\
(2017)\end{array}$ & $\begin{array}{l}\text { Reino } \\
\text { Unido }\end{array}$ & $\begin{array}{l}1151 \text { mães, } 412 \text { BLW } \\
\text { estrito; } 377 \text { BLW solto } \\
\text { e } 362 \mathrm{MT}\end{array}$ & $\begin{array}{l}\text { Método tradicional } \\
\text { e BLW }\end{array}$ & $\begin{array}{l}\text { Episódios de engasgo: } \\
11,9 \% \text { BLW estrito } \\
15,5 \% \text { BLW solto } \\
11,6 \% \text { MT }\end{array}$ \\
\hline $\begin{array}{l}\text { Daniels et al. } \\
(2018)\end{array}$ & $\begin{array}{l}\text { Nova } \\
\text { Zelândia }\end{array}$ & $\begin{array}{l}206 \text { participantes, } 101 \\
\text { Controle e } 105 \text { BLISS }\end{array}$ & $\begin{array}{l}\text { Método tradicional } \\
\text { e BLISS }\end{array}$ & $\begin{array}{l}\text { Ingesta de Zinco (mg/dia) } \\
\text { - Aos } 7 \text { meses: } \\
\text { Controle: } 3,5 ; \text { BLISS: } 3,5 \\
\text { - Aos } 12 \text { meses: } \\
\text { Controle: } 4,4 \text { BLISS: } 4,4\end{array}$ \\
\hline
\end{tabular}

Fonte: Dados da pesquisa (2020).

A Tabela 1 faz uma síntese das características dos trabalhos analisados na presente revisão, apresenta o nome dos autores e ano de publicação, o país onde o estudo foi realizado, a amostra analisada, quais os métodos de introdução alimentar abordados e os principais resultados.

No estudo de Taylor et al (2017) com 206 crianças, cuja a triagem ocorreu no período final da gestação de suas mães, 101 crianças fizeram parte do grupo controle, o qual realizava o método de introdução alimentar tradicional e 105 do BLISS. As crianças tiveram os dados antropométricos e comportamentais avaliados desde o nascimento até completar 24 meses. Todas as mães foram orientadas a iniciarem a introdução alimentar apenas após os bebês completarem 6 meses. Na avaliação do IMC a diferença percentual entre o grupo controle e o BLISS foi menor que 1\%, nas avaliações realizas aos 12 meses e aos 24 meses. No quesito consumo energético foi analisado aos 7, 12 e 24 meses, sendo aos 7 meses uma diferença de consumo 
energético entre o grupo controle e o BLISS de apenas $134 \mathrm{KJ}$ e aos 12 e 24 meses menor que $60 \mathrm{KJ}$. Na análise dos dados observa-se que não há diferenças em termos energéticos e antropométricos que possam interferir no crescimento e desenvolvimento de um bebê saudável.

O trabalho realizado Morison et al.(2016) avaliou crianças de 6 a 8 meses, os cuidadores responderam a um questionário demográfico e um questionário alimentar, além disto as crianças tiveram suas dietas pesadas. $\mathrm{O}$ trabalho contou com 26 participantes no grupo controle, que realizou o método de introdução alimentar tradicional e 25 participantes do método BLW, dos quais 7 realizavam o método parcial e 18 o total.

No trabalho de Morison et al. (2016) para realizar o cálculo dos nutrientes ingerido pelas crianças foi utilizado o registro da dieta fornecido através do questionário. Dentre os nutrientes avaliados no trabalho baseado na dieta de uma criança de 7 meses, observou-se que as crianças em BLW parcial consumiram uma média de $3073 \mathrm{KJ}$, porém em nenhuma dos três métodos o consumo estava abaixo do valor de referência que foi de $2800 \mathrm{KJ}$, para os meninos e 2500 para as meninas. O consumo de proteína, gordura total, zinco, cálcio e sódio nos três métodos avaliados no estudo estavam dentro dos valores de referência sem diferença significativa entre os mesmos. Já quanto ao consumo de ferro e Vitamina $\mathrm{B}_{12}$ o método BLW total apresentou valores abaixo do esperado, sendo o valor da ingesta de ferro menor que o valor de referência em todos os métodos. Em valores absolutos apresentados pela pesquisa, destaca-se que não há aumento dos riscos na ingesta de nutrientes ou benefícios, baseados no tipo de método de introdução alimentar. Porém, destaca-se que há uma deficiência de nutrientes como ferro e vitamina $B_{12}$ na dieta de forma geral da criança.

O estudo prospectivo randomizado de Dogan et al (2018) contou com 280 bebês, do quais 142 seguiam o BLW e 138 o método tradicional. Na pesagem aos 12 meses de idade o peso médio em quilogramas no grupo BLW era de 10,4 e no grupo do método tradicional era de 11,1, observando-se assim um peso maior dos bebês em método tradicional. Seguindo os padrões de escore z orientados pela OMS o grupo BLW tinham $98 \%$ dos bebês com peso normal e $2 \%$ com baixo peso, já os do método tradicional $83 \%$ apresentou peso normal e 17\% sobrepeso. Demonstrou assim que o grupo que seguiu a abordagem tradicional ganhou mais peso, apesar das diferenças antropométricas não serem significativas, além disto os bebês que seguiram o BLW demonstraram uma tendência maior de regulação energética, acredita-se que pelo fato de que no método tradicional são os pais e /ou cuidadores que possuem maior controle sob a alimentação. Observa-se que o método tradicional mostrou maior risco para o desenvolvimento da obesidade, acredita-se que decorre do fato de o BLW permitir que a criança exerça o controle sobre a sua saciedade.

No mesmo trabalho de Dogan et al.,(2018) foram avaliados padrões hematológicos como hemoglobina, hematócrito, RDW, VCM, ferritina e saturação de ferritina, que ao nascer e após os 6 meses de intervenção não apresentaram diferenças significativas entre os grupos. Segunda a observação do registro de dieta, a ingestão de ferro aos 12 meses era semelhante entre os grupos, sendo a média do BLW de 7,97 mg/dia e do grupo do método tradicional de 7,90 mg/dia.Vale ressaltar que apesar de não haver diferença relevante entre os grupos quanto a ingesta de Ferro, nenhum dos dois estavam dentro dos padrões estabelecidos pela OMS para o consumo de Ferro que é de 10,8 mg/dia. Porém na avaliação hematológica aos 12 meses nenhum dos grupos tinham bebês anêmicos

No estudo realizado por Dogan et al., (2018) foi feita uma observação quanto ao período do fim do aleitamento exclusivo pelas mães, observando-se que mães que optaram pelo BLW iniciou a introdução alimentar pelo menos 1 semana depois que as mães do método tradicional, além disto as mães que realizaram o BLW ofertaram alimento sólido aos seus bebês mais cedo que as mães do método tradicional. A diferença entre início de introdução alimentar entre os grupos revelou uma maior tendência das mães do grupo BLW a amamentar exclusivamente até os 6 meses 
Outro aspecto avaliado por Dogan et al., (2018) foi quanto o risco de engasgo, para tanto foi questionado as mães e aos cuidadores quanto a frequência de engasgo semanalmente, o grupo BLW apresentou 2 episódios e o tradicional 3, demonstrando que não há riscos elevados devido ao tipo de método alimentar.

O trabalho de Cameron et al., (2015) aborda a adequação da ingesta de Ferro, ingesta energética e risco de asfixia através de um comparativo entre o BLW e o BLISS, avaliado em crianças de 6 a 8 meses. Os dados foram obtidos através de entrevistas realizadas por doze semanas e questionário com a oferta alimentar. Ao total foram 23 participantes, 14 do método BLISS e 9 do BLW. A quantidade de Ferro presente nos alimentos ( $\mathrm{mg} / \mathrm{dia}$ ) (de acordo com os registros de dieta de 3 dias) foi para o BLISS (4,9 mg / dia) e BLW (2,2 mg / dia). De acordo com o estudo não foi considerada uma diferença significativa. Já em relação a ingesta de proteína, por gramas de carne vermelha oferecida por dia foram significativamente maiores no BLISS (20,1 g / dia) quando comparado ao grupo BLW (3,2 g / dia). A quantidade de energia oferecida na alimentação complementar ( $\mathrm{kJ} / \mathrm{dia}$ ) (de acordo com os registros de dieta de 3 dias) foi para o BLISS (2228 kJ / dia) e para o BLW (1862 kJ / dia), considerada uma diferença não significativa para o estudo. A incidência de engasgo foi de 2 episódios no grupo BLISS e 1 no grupo BLW, demonstrando que não há aumento dos riscos pelo tipo de método de introdução alimentar

Erickson et al., (2018) realizou ensaio clínico randomizado avaliando os tipos de alimentos e os nutrientes ingeridos por bebês através da avaliação de um grupo controle e grupo BLISS. Os dados foram obtidos quando os bebes tinham 7, $12 \mathrm{e}$ 24 meses, levando também em consideração a dieta total, ou seja, leite ou fórmula mais a alimentação complementar. Os nutrientes avaliados no estudo foram: energia, proteína, gordura total, gordura saturada, carboidratos, fibra dietética, vitamina C, vitamina $\mathrm{B}_{12}$, cálcio e sódio. Dentre as principais mudanças constou-se que: aos 7 meses os bebes do método BLISS consumiam mais gordura total e sódio que os bebês do grupo controle, sendo os valores de consumo do sódio no grupo BLISS $301 \mathrm{mg}$ e no grupo controle $223 \mathrm{mg}$, aos 12 meses os bebes BLISS consumiram 7\% a menos de gordura saturada que os do grupo controle e aos 24 meses todos os nutrientes tiveram valores semelhantes. O presente estudo demonstrou que a diferença entre os métodos de introdução alimentar não impactou de forma significativa nos valores nutricionais ingeridos pelos bebês.

No estudo realizado por Daniels et al., (2018) contou com 206 participantes, 101 faziam parte do grupo controle e 105 realizaram o método BLISS, ao longo da avaliação houve redução de participantes em ambos os grupos. O consumo energético foi calculado em ambos os grupos, aos 7 meses os grupos apresentaram uma diferença de $134 \mathrm{KJ}$, já aos 12 meses a diferença foi ainda menor, $50 \mathrm{KJ}$. Foram avaliadas também a ingesta de Ferro e outros nutrientes com base nos registros da dieta pesada de 3 dias quando os bebes tinham 7 e 12 meses e aos 12 meses foi realizada avaliação sérica.

Quanto a ingesta de Ferro aos 7 e 12 meses a maior diferença apresentada entre o grupo BLISS e o grupo controle foi de $0,6 \mathrm{mg} /$ dia. Já no cálculo da ingesta de vitamina C o grupo BLISS apresentou um valor de 49,2 $\mathrm{mg} / \mathrm{dia}$ e os bebes do grupo controle 59,2 mg/dia, observando-se assim um valor menor para o grupo BLISS. De forma geral, ambos os grupos da pesquisa apresentavam uma ingesta inadequada de ferro aos 7 meses, chegando a 74\% para ambos os grupos, diferença que reduziu aos 12 meses, $23 \%$ do grupo controle e $26 \%$ do grupo BLISS.

Além dos valores baseados na pesagem da dieta, aos 12 meses os bebes passaram por uma avaliação laboratorial através da coleta de sangue. Os valores para hemoglobina (g/dl) no grupo controle foi de 11,7 e no grupo BLISS 11,6. Quanto a dosagem de Ferro a maioria das crianças em ambos os grupos apresentou valores satisfatórios, apenas 5\% do grupo controle e 7\% do grupo BLISS apresentou anemia por deficiência de ferro. Houve ainda uma diferença média de 2,6ug/L entre o grupo BLISS e o grupo controle para ferritina plasmática, não sendo significativa. Quanto aos demais indicadores bioquímicos para avaliação do estado de ferro, não apresentaram valores estatisticamente significantes. De acordo com os dados obtidos no estudo não há diferença na ingesta de ferro para bebes que seguem a alimentação por colher e as que seguem o BLW modificado (BLISS). Portanto neste aspecto a avaliação deve ser cautelosa, pois ouve uma modificação do método BLW com vistas a reduzir a deficiência de Ferro. 
Em outro estudo realizado por Daniels et al., (2018) avaliou-se a ingestão, fontes e o status bioquímico de zinco em bebes que realizam o método BLISS em relação ao tradicional. A avaliação foi realizada aos 7 e 12 meses por meio de registro da dieta pesada de 3 dias e concentração plasmática do zinco aos 12 meses. A ingesta de zinco mg/dia aos 7 meses foi igual para o grupo controle e o grupo BLISS, $3,5 \mathrm{mg} / \mathrm{dia}$, levando em consideração o leite e os alimentos complementares. Aos 12 meses a ingesta de zinco $\mathrm{mg} / \mathrm{dia}$ foi de 4,4 $\mathrm{mg} /$ dia para ambos os grupos também. Na avaliação plasmática realizada aos 12 meses o grupo controle apresentou $36 \mu \mathrm{g} / \mathrm{dL}$ e o grupo BLISS $33 \mu \mathrm{g} / \mathrm{dL}$, demonstrando que não há influencia na ingestão de zinco baseada no tipo de método de introdução alimentar.

Dos 8 trabalhos analisados, 3 abordaram a ocorrência de engasgo entre os diferentes métodos. O que apresentou maior amostragem foi o realizado por Brow A. (2017), no qual 1151 mães responderam a um questionário. Através das respostas obtidas, 412 se classificaram como BLW estrito, 377 BLW solto e 362 método tradicional. Dentre essa classificação, 155 bebes dos 1151 teve ao menos um episódio de asfixia, sendo 11,9\% do grupo BLW estrito, 15,5\%\% do grupo BLW solto e 11,6\% do grupo tradicional. Os resultados obtidos nos outros dois estudos foram, em um, 2 episódios de engasgo no grupo BLW e 3 no método tradicional e o outro, 2 episódios no grupo BLISS e 1 no grupo BLW ((Dogan et al.,(2018); Cameron et al., (2015)). Analisa-se que mesmo com diferentes tamanhos de amostras e os estudos abordando diferentes métodos, nenhum dos resultados obtidos demonstrou diferenças significativas para o risco de engasgo.

\section{Conclusão}

Está revisão não identificou diferenças significativas entres os diferentes métodos de introdução alimentar. Apenas um dos trabalhos avaliou o impacto no desenvolvimento, concluindo que não há impacto sob o mesmo. Conclui-se também que dentre os métodos não há nenhum que aumente ou reduza os riscos de deficiências nutricionais que gerem impactos na saúde. Apenas um dos estudos observou maior taxa de bebês sobrepeso no método tradicional, alertando para necessidade de mais estudos que avaliem os riscos de obesidade de acordo com o método de introdução alimentar. No que diz respeito a segurança dos métodos alimentares, quanto ao risco de engasgo, dos três estudos que avaliaram tal aspecto, nenhum deles observou um método que leve a maiores riscos

\section{Referências}

Andries e Arantes, A. L., Neves F. S., Campos A. A. L., \& Netto M. P.(2018). The baby-led weaning method (BLW) in the context of complementary feeding: A review. Revista Paulista de Pediatria, 36(3), 353-363.

Brown, A. (2017) No difference in self-reported frequency of choking between infants introduced to solid foods using a baby-led weaning or traditional spoonfeeding approach.

Cameron, S. L.; Taylor, R. W.; \& Heath, A.-L. M. (2015). Development and pilot testing of Baby-Led Introduction to SolidS-a version of Baby-Led Weaning modified to address concerns about iron deficiency, growth faltering and choking.

Daniels, L. et al. (2018). Impact of a modified version of baby-led weaning on iron intake and status: a randomised controlled trial. BMJ Open,8, 19036.

Daniels, L. et al. (2018) Modified Version of Baby-Led Weaning Does Not Result in Lower Zinc Intake or Status in Infants: A Randomized Controlled Trial. $J$ Acad Nutr Diet,1006-1016.

De-la-Torre-Ugarte-Guanilo, M. C., Takahashi, R. F., \& Bertolozzi, M. R. (2011). Revisão sistemática: noções gerais.

Dogan, E., et al. (2018). Baby-led complementary feeding: Randomized controlled study. Pediatrics International,1073-1080.

Erickson, L. W., et al. (2018). Impact of a Modified Version of Baby-Led Weaning on Infant Food and Nutrient Intakes: The BLISS Randomized Controlled Trial. Nutrients, 10,740 .

Fangupo, L. J., et al. (2016) A baby-led approach to eating solids and risk of choking. Pediatrics, 138(4).

Lima, D. B. et al.(2012). Alimentação na primeira infância no Brasil. Revista de APS, 15(3).

Lopes, W. C. et al. (2018) Alimentação de crianças nos primeiros dois anos de vida. Rev. paul. pediatr,164-170. 
Research, Society and Development, v. 10, n. 4, e44110414290, 2021

(CC BY 4.0) | ISSN 2525-3409 | DOI: http://dx.doi.org/10.33448/rsd-v10i4.14290

Monte, C. M. G., \& Giugliani, E. R. J. (2004.) Recomendações para alimentação complementar da criança em aleitamento materno. J Pediatr, 80(5), 131-141.

Morison, B. J. et al. (2016). How different are baby-led weaning and conventional complementary feeding? A cross-sectional study of infants aged 6-8 months. BMJ Open, 6, 10665 .

Rapley G., Tracey M. (2008). Baby-led weaning. Essence; 44:1

Rapley G. (2015). Baby-led weaning: The theory and evidence behind the approach. Journal of Health Visiting, 3(3), 144-151.

Sankar, M. J., Sinha, B., Chowdhury, R., Bhandari, N., Taneja, S., Martines, J., et al. (2015). Optimal breastfeeding practices and infant and child mortality: a systematic review and metaanalysis. Acta Paediatr. 104(467),3-13.

SBP. Sociedade Brasileira de Pediatria. (2012). Manual de orientação para a alimentação do lactente, do pré-escolar, do escolar, do adolescente e na escola/Sociedade Brasileira de Pediatria. Departamento de Nutrologia, (3a. ed.).

SBP. Sociedade Brasileira de Pediatria. (2017). Guia Prático de Atualização. A Alimentação Complementar e o Método BLW (Baby-Led Weaning). Departamento de Nutrologia.

Sorrentino, E. (2018). Alimentação complementar: barreiras e facilitadores sob a ótica de cuidadores. Projeto de dissertação de mestrado. Faculdade de Saúde Pública São Paulo: da USP, São Paulo, SP, Brasil.

Taylor, R. W., et al. (2017) Effect of a Baby-Led Approach to Complementary Feeding on Infant Growth and Overweight A Randomized Clinical Trial Supplemental content. JAMA Pediatr, 171(9), 838-846.

Victora, C. G., Bahl, R., Barros, A. J. D., França, G. V. A., Horton, S., \& Krasevec, J. (2016) Breastfeeding in the 21st century: epidemiology, mechanisms, and lifelong effect. The Lancet, 387.

WHO. (2001). Global Consultation on Complementary Feeding. Guiding Principles for Complementary Feeding of the Breastfeed. 